\title{
Moyens de recouvrement du brûlé grave : réflexions sur leur application aux lésions radiologiques
}

\author{
H. CARSIN ${ }^{1}$, J. STÉPHANAZZI ${ }^{1}$, F. LAMBERT ${ }^{2}$, P.-M. CURET ${ }^{3}$, \\ P. GOURMELON ${ }^{4}$
}

(Manuscrit reçu le 30 mai 2000 , accepté le 16 juillet 2001 )

R ÉSUMÉ Après une brûlure grave, le blessé est fragilisé par le risque infectieux et une réaction inflammatoire générale. Les tissus nécrosés doivent être enlevés car ils sont toxiques pour l'organisme. Il faut donc restituer au malade une enveloppe cutanée, ce qui est le rôle d'un centre de traitement des brûlés. I.es différentes techniques possibles sont décrites :

- autogreffes conventionnelles sur les brûlures peu étendues ;

- substituts cutanés pour recouvrir temporairement les brûlures étendues. Parmi ceux-ci on distingue :

- les substituts naturels : xénogreffes (peau de porc...) ou allogreffes (peau humaine),

- les substituts naturels transformés qui ne conservent que la trame de la matrice extra cellulaire. Les peaux artificielles appartiennent à cette catégorie et permettent d'obtenir des cicatrices de grande qualité,

- Ies cultures cellulaires qui consistent en la multiplication en laboratoire des cellules d'un individu qui lui seront greffées ensuite. Ce n'est pas encore une technique de routine mais elle peut permettre la survie de malades très graves.

Les brûlures radiologiques soulèvent beaucoup de questions. Leurs caractéristiques sont analysées: Douleur intense, permanente, résistante aux antalgiques, parallélisme difficile à établir avec les brûlures thermiques...

Malgré le peu de cas rencontrés, quelques réflexions et orientations sur la prise en charge des radionécroses peuvent être données : importance du confort du malade, de la sédation des douleurs, de la prévention de l'infection et de l'équilibre nutritionnel. Au stade de l'épidermite et des phlyctènes, le traitement peut être complété par l'utilisation de facteurs de croissance. Au stade de nécrose, après couverture temporaire, l'autogreffe ne doit intervenir que si on est sûr de la nonévolutivité du socle receveur. L'indication des cultures cellulaires pour apporter harmonieusement des facteurs de croissance peut être discutée.

ABSTRACT Covering techniques for severe burn treatment: lessons for radiological burn accidents.

After a severe burn, the injured person is weakened by a risk of infection and a general inflammation. The necrotic tissues have to be removed because they are toxic for the organism. The injured person also needs to be covered by a cutaneous

\footnotetext{
1 Centre de traitement de brûlés, HIA Pcrcy, 92140 Clamàt, France.

${ }^{2}$ Service de chirurgie plastique, HIA Percy, 92140 Clamar, France.

${ }^{3}$ Service de protection radiologique des armées, rue Lt. R. Balamy, 92 I 40 Clamart, Frante.

${ }^{4}$ Institut de protection el de sûreté nucléairc, BP 6, 92265 Fontendy-aux-Roses, Firance.
} 
envelope, which has to be done by a treatment centre for burned people. The different techniques are the following:

- autografts on limited burned areas;

- cutaneous substitutes to cover temporary extended burned areas. Among them:

- natural substitutes like xenografts (pork skin, sheep skin,..) or allografts (human skin),

- treated natural substitutes which only maintain the extracellular matrix. Artificial skins belong to this category and allow the development of high quality scars,

- cell cultures in the laboratory: multiplying the individual cells and grafting them onto the patient. This technique is not common but allows one to heal severely injured patients.

X-ray burns are still a problem. Their characteristics are analysed: intensive, permanent, antalgic resistant pain. They are difficult to compare with heat burns. In spite of a small number of known cases, we can give some comments and guidance on radio necrosis cures: the importance of the patient's comfort, of ending the pain, of preventing infection, and nutritional balance. At the level of epidermic inflammation and phlyctens (skin blisters), the treatment may be completed by the use of growth factors. At the level of necrosis, after a temporary cover, an autograft can be considered only if a healthy basis is guaranteed. The use of cellular cultures in order to obtain harmonious growth factors can be argued.

Les brûlures radio-induites restent bien mystérieuses pour le spécialiste des brûlés lorsqu'il les compare aux autres types de lésions : mystérieuses par leur aspect, mystérieuses par leur évolution. Il est difficile d'en proposer une thérapeutique adaptée sans que ces mystères soient levés.

Le but d'un centre de traitement des brûlés est de restituer au blessé qui l'a tout ou partiellement perdue une enveloppe cutanée. Cette habitude de prise en charge du «malade sans peau » en fait un lieu privilégié pour le traitement des lésions cutanées radiologiques par le support de réanimation et les techniques chirurgicales qui y sont employées.

Plutôt que de vous proposer un schéma de traitement dogmatique, figé et sans doute erroné, il m'a paru plus intéressant de développer devant vous les différents moyens de recouvrement actuellement employés dans la couverture des brûlés graves puis de réfléchir à leurs éventuelles indications dans la thérapeutique du blessé radiologique.

\section{Le recouvrement du brûlé grave}

\subsection{Le tissu brûlé est toxique}

Le tissu brûlé est capable d'entraîner la mort de l'animal de laboratoire par le biais d'une toxine identifiée. Celle-ci est le point de départ d'une réaction inflammatoire aiguë comme le sont les toxines microbiennes. 
À la place de la peau se développe une plaie, brèche où vont s'accumuler plaquettes puis leucocytes puis macrophages. La réaction inflammatoire ainsi développée s'exporte lorsqu'elle atteint une grande masse de tissus : les cytokines agissent à distance et interférent dans la régulation thermique, le métabolisme et l'équilibre endocrinien du patient. Elle aboutit rapidement à une dépression immunitaire d'autant plus dangereuse que le blessé est « ouvert » à l'infection. Celle-ci, lorsqu'elle survient, aggrave la réaction générale, diminue les défenses et favorise une nouvelle infection, générant un cercle vicieux qui peut emporter le brûlé...

Il est donc important d'enlever le plus vite possible les tissus nécrosés qui ne cicatriseront pas spontanément et sont un lieu propice à l'accumulation bactérienne, peu ou pas accessible aux antibiotiques. Ce geste d'excision, d'autant plus urgent que la brûlure est plus grave, est un geste très agressif : il consiste à enlever les tissus brûlés en s'arrêtant le plus souvent à l'aponévrose musculaire.

Les zones ainsi découvertes doivent être immédiatement recouvertes !

\subsection{Les autogreffes conventionnelles}

Lorsque la brûlure est limitée en surface, la peau saine du malade est prélevée au rasoir et transposée sur la zone excisée : c'est le principe de l'autogreffe. Afin d'en augmenter la surface, la bande de peau prélevée est transformée en un filet aux mailles plus ou moins larges (technique du mesh-graft).

Pour des brûlures très étendues, il faut recourir à une solution temporaire qui permet de recouvrir les zones excisées pendant que les prises de greffe cicatrisent (et puissent être prélevées une seconde voire une troisième fois) ou que les lésions superficielles guérissent servant à leur tour de zones donneuses.

\subsection{Qu'est-ce qu'un substitut cutané?}

Les produits destinés à remplacer de façon provisoire ou définitive sont légions.

Le substitut cutané idéal a les propriétés suivantes : il prévient la dessiccation de la plaie et diminue les pertes de chaleur et l'évaporation, il empêche la contamination microbienne et limite les pertes sanguines et protidiques, il diminue la douleur. Placé sur des brûlures superficielles ou intermédiaires, il maintient une atmosphère propice à la cicatrisation qu'il peut accélérer ; placé sur des brûlures profondes, il prépare le socle à la greffe.

On peut à l'heure actuelle classer les substituts cutanés en :

- substituts naturels : xénogreffes, membrane amniotique, allogreffes,

- substituts naturels transformés : à partir de xénogreffes ou d'allogreffes, 
- substituts semi-synthétiques à base de collagène et de composés synthétiques : les peaux artificielles,

- composés semi-synthétiques contenant des cellules allogéniques,

- cultures de peau.

\subsection{Les xénogreffes}

Elles constituent une source importante et intéressante de substituts cutanés. Employées à grande échelle dans certains pays comme la Chine, il s'agit essentiellement de peau de porc. En pays musulman, la peau de mouton a été utilisée, comme au Brésil la peau de grenouille. En l'absence de traitement, ces xénogreffes posent le problème de la transmission à l'homme de virus ou d'agents infectieux non-conventionnels. On utilise donc de plus en plus souvent des xénogreffes traitées qui sont décellularisées (EZ-DERM ${ }^{\mathrm{TM}}$ ) mais dont a gardé la trame. Ces xénogreffes se comportent comme des pansements biologiques et ne s'intègrent pas au socle receveur.

\subsection{Les allogreffes}

Il s'agit donc de peau humaine. En France, les allogreffes cutanées sont prélevées plus souvent dans le cadre d'un prélèvement multi-organes que sur un cadavre dans les 8 heures qui suivent sa mort.

La conservation se fait par cryopréservation au niveau d'une banque de tissus qui en assure la sécurisation et la traçabilité. Les allogreffes fraîches permettent une couverture de meilleure qualité mais sont quasi impossibles à obtenir en grande quantité en urgence. Le traitement des allogreffes par ultraviolets permet d'en améliorer la survie. Le risque de transmission de virus (CMV ou HIV) existe.

Il existe en France une grande pénurie en allogreffes cutanées, elles restent pourtant «le gold standard» des substituts cutanés, indispensables comme couverture temporaire en l'attente de cultures cutanées. L'utilisation d'allogreffes d'importation (Euroskin Bank ${ }^{\mathrm{TM}}$ en Hollande) est donc de plus en plus fréquente.

Les allogreffes conservées dans du glycérol à $85 \%$ (qui tuent les éléments cellulaires) présentent un risque moindre de transmission virale.

Quel que soit leur mode de conservation, les allogreffes sont mises en place sur le lit fraîchement excisé, il s'y développe un bourgeon vasculaire, reperméabilisation des vaisseaux de l'allogreffe par des vaisseaux venant du receveur. Ces allogreffes sont rejetées à partir de la $3^{\mathrm{e}}$ semaine suivant leur mise en place. 
Dans de très rares cas, ces allogreffes fraîches ont été utilisées comme couverture définitive avec l'appoint de médicaments anti-rejets.

\subsection{Substituts naturels transformés}

Comme nous l'avons déjà évoqué à propos de l'EZ-DERM ${ }^{\mathrm{TM}}$ qui rentre dans le cadre de ces substituts naturels transformés, il apparaît intéressant de conservée la trame de la matrice extra-cellulaire et de la jonction dermo-épidermique en enlevant les cellules. L'ALLODERM ${ }^{\mathrm{TM}}$ est ainsi un substitut cutané constitué d'une allogreffe humaine traitée par des détergents et décellularisée. Son utilisation comme support dermique permet d'améliorer l'aspect cosmétique et mécanique. Il doit cependant être recouvert dans le même temps d'une autogreffe très fine et ne présente alors qu'un intérêt très limité dans le traitement des lésions de grande surface. Employé dans le comblement de pertes de substances importantes, il a un intérêt tout autre. Ce produit n'est pas encore importé en France.

\subsection{Les peaux artificielles}

On réserve cette dénomination à des composés semi-synthétiques bilaminaires (qui possèdent donc un derme et un épiderme).

\section{Le Biobrane ${ }^{\mathrm{TM}}$}

Il est le plus ancien de ces substituts : l'épiderme est fait de silicone, le derme de collagène porcin de type I enrobé dans un filet de Nylon. Mis en place après excision sur un socle exsangue et stérile, c'est une membrane élastique et transparente qui peut jouer un rôle de couverture cutanée temporaire (de moindre qualité que les allogreffes humaines). Non biodégradable, il doit être enlevé avant greffes définitives. Il n'est pas sur la liste des produits autorisés à l'importation (faute de l'avoir demandé). Outre Atlantique, ce produit est le plus souvent utilisé comme pansement des brûlures superficielles dont il favorise la cicatrisation avec un excellent confort pour le patient.

\section{L’Integra $^{\mathrm{TM}}$}

Peau artificielle mise au point par Yannas et Burke en 1971 (Burke et al., 1981), c'est le produit le plus novateur : l'épiderme est une membrane synthétique de silicone dont la perméabilité est contrôlée, le derme est une matrice de porosité particulière faite de fibres entrecroisées de collagène de tendons de veau et de chondroïtine-6-sulfate de requin. Cette peau se conserve dans de l'alcool à $70^{\circ}$ au réfrigérateur et est donc immédiatement disponible. 
L'Integra est mis en place après excision, il réalise une très bonne couverture du patient. La matrice de collagène sert d'échafaudage à la migration des cellules et des néovaisseaux qui migrent à partir du socle receveur. Elle génère en 2 à 3 semaines un néoderme de qualité, peu inflammatoire et bien architecturé. Passé ce délai, le silicone est enlevé et remplacé par une autogreffe prise en zone saine posée sur le néoderme. Cette autogreffe peut être très fine, limitant les cicatrices des zones de prise ou permettant une reprise plus précoce.

La cicatrice de greffes obtenues est de qualité : moins inflammatoire, moins hypertrophique, moins rétractile et beaucoup plus souple. Les éléments du derme artificiel sont biodégradables et disparaissent en quelques mois.

Couverture immédiate et cicatrice de qualité, tels sont donc les avantages de ce produit qui permet ainsi de traiter les brûlés graves. Le derme obtenu n'est, à l'heure actuelle, pas apte à nourrir et ancrer une culture cellulaire et doit donc toujours être recouvert d'une autogreffe expansée.

Utilisé sur de lésions couvrant de 40 à $60-70 \%$ de la surface corporelle, il nécessite des surfaces donneuses en quantité suffisante. La qualité fonctionnelle des cicatrices obtenues en fait un recouvrement de choix pour les brûlures profondes en zone articulaire et, peut-être, sur le visage ou chez les patients à haut risque de cicatrice hypertrophique (peaux noires).

\section{Le Transcyte ${ }^{T M}$}

Il est le premier produit d'une nouvelle classe de substituts cutanés, les substituts cutanés semi-synthétiques contenants des cellules allogéniques. Il s'agit d'une peau artificielle à base de BiobraneTM dans laquelle ont été incorporés des fibroblastes humains cultivés à partir de prépuces de nouveau-nés. Bien évidemment, les souches de fibroblastes sont étroitement contrôlées et une traçabilité mise en place. La conservation du produit est également contraignante (dans un liquide nutritif spécial à moins $24^{\circ} \mathrm{C}$ ).

Le substitut est mis en place après excision, il permet la couverture temporaire du brûlé et facilite le développement d'un derme apte à recevoir dans un second temps une autogreffe fine. L'ablation de la peau artificielle non biodégradable en est évidemment le préalable. La présence de cellules vivantes favorise la cicatrisation. Une autre indication de ce produit apparaît donc, elle consiste à l'utiliser sur de brûlures superficielles ou intermédiaires afin d'en accélérer la guérison.

\subsection{Les cultures cellulaires}

Il s'agit de la multiplication en laboratoire des cellules d'un individu, le tissu obtenu lui étant ensuite greffé. Sur le plan immunologique, il existe une grande 
différence entre la principale cellule de l'épiderme, le kératinocyte qui est étroitement spécifique de l'individu et capable de déclencher une réaction de rejet de greffe et la cellule principale du derme, le fibroblaste, qui est immunitairement bien plus compliant. La culture d'épiderme est actuellement la plus utilisée en couverture du brûlé grave.

\section{Les cultures d'épiderme}

Rheinwald et Green (1975) ont découvert la possibilité de cultiver des kératinocytes sur un support nutritif constitué de fibroblastes murins malins irradiés de façon létale, le tout dans un milieu de culture à la composition parfaitement définie.

Les kératinocytes mis en culture proviennent d'une à deux biopsies de 2 à $4 \mathrm{~cm}^{2}$ réalisées sur le malade au niveau d'un creux axillaire ou d'un pli inguinal. Chaque kératinocyte ensemencé forme une colonie. En 8 jours, les colonies sont confluantes, c'est la fin de la culture primaire. Chaque culture primaire est remise en culture pour donner à son tour une dizaine de cultures secondaires qui peuvent à leur tour être remises en culture... Le pouvoir d'expansion est considérable : à partir d'un $\mathrm{cm}^{2}$ de biopsie, on peut disposer de $1 \mathrm{~m}^{2}$ de cultures dans un délai de 18 jours. Ce sont, en général, les cultures secondaires qui sont greffées.

Le jour de la greffe, chaque fragment d'épiderme se présente comme un tissu transparent, non-manipulable car trop fragile et polarisé (fait de 4 à 8 couches cellulaires dont l'une basale). Il est décollé de son support, installé et agrafé sur une gaze et placé dans un milieu et une boîte de transport, elle-même placée dans un conteneur spécial. La culture doit être utilisée dans les 24 heures suivantes. Chaque greffon mesure $50 \mathrm{~cm}^{2}$ (25 auparavant). Ce travail est très long, nécessite un personnel compétent en nombre dès lors que la surface à greffer est importante (en moyenne à Percy 210 greffons de $25 \mathrm{~cm}^{2}$ par pose).

Pendant les 2 à 3 semaines de pousse des cultures, le malade doit être maintenu en vie, exempt de toute colonisation microbienne, dans un état nutritionnel apte à cicatriser. Les brûlures profondes sont toutes excisées et le malade recouvert d'allogeffes.

Lorsque les cultures arrivent, les chirurgiens enlèvent par dermabrasion la partie superficielle, épidermique, des allogreffes (celles-ci doivent donc adhérer au socle receveur). C'est sur le néoderme ainsi obtenu que les greffons d'épiderme sont posés, agrafés et recouverts d'un tulle perméable aux exsudats (voile de mariée) qui solidarise le tout. 
Sept jours après la pose, le voile de mariée et la gaze support sont enlevés, c'est le «take-down ». On peut alors juger du degré de prise, taux d'épidermisation des zones greffées. Par la suite, en fonction de la qualité des cultures, de l'état du patient et de la qualité des soins locaux, cette épidermisation peut s'étendre ou, au contraire les cultures peuvent lyser.

La charge de soins est énorme : 90 minutes de pansement quotidien pour 4 à 6 personnes. La cicatrice obtenue est fragile pendant longtemps, elle est peu esthétique et rétracte de façon importante. Le coût est majeur, $100 \mathrm{~F}$ par $\mathrm{cm}^{2}$ pour les seules cultures. Le vieillissement des cellules cultivées et leur avenir à long terme est encore mal connu: les conditions de culture influent sur ce vieillissement. Il est donc impossible aujourd'hui de présenter cette technique comme de routine. Elle permet néanmoins des sauver des malades qui, sans elle, sont irrémédiablement condamnés.

Les problèmes cicatriciels de ces cultures d'épiderme autologue sont liés à l'immaturité de la jonction dermo-épidermique. Cette jonction qui permet l'ancrage de l'épiderme dans le derme est constituée pour $70 \%$ d'éléments fournis par les kératinocytes et pour $30 \%$ d'éléments fournis par les fibroblastes. Il apparaît donc essentiel de s'intéresser aux fibroblastes dont la plasticité se révèle moindre qu'on ne l'avait imaginé afin de fournir aux cultures d'épiderme un socle de meilleure qualité. C'est l'enjeu des années à venir : proposer aux patients une couverture immédiate d'un derme qui serait ensuite recouvert de cultures d'épiderme.

Des progrès sont également attendus dans la qualité des cultures d'épiderme : sélection des kératinocytes mis en culture au profit des cellules à haut potentiel clonogènique, cultures secondaires réalisées sur un support qui permet d'éviter un décollement enzymatique fragilisant et une rétraction (cultures sur colle biologique).

\section{Les autres types de cultures de peau}

Différents modèles de cultures de peau sont en cours de développement au stade de la recherche clinique.

Les cultures comprenant à la fois derme et épiderme, kératinocytes et fibroblastes, pourraient avoir des développements thérapeutiques car ils ont l'avantage de fournir une jonction dermo-épidermique mature :

- le modèle de Bell modifié par Coulomb et Dubertret (Coulomb et al., 1998) consiste en un derme équivalent obtenu par rétraction d'un gel de collagène par des fibroblastes. De petits morceaux de peau (contenant des kératinocytes) sont ensemencés à la surface de ce derme équivalent. Ce modèle a été utilisé dans 
la couverture de naevi géants mais les résultats obtenus chez le brûlé grave se sont avérés décevants ;

- le modèle de Boyce et Hansbrough (Boyce et al., 1995) est obtenu à partir d'une membrane de collagène et glycosaminoglycanes proche de l'Integra. Y sont successivement ensemencés fibroblastes puis kératinocytes. Ce type de cultures a pu être placé avec succès sur de l'Integra. Un modèle voisin est en cours de développement en France sur un derme contenant de la chitosine.

\section{L'utilisation des cultures de peau}

Actuellement nous utilisons les cultures d'épiderme obtenues selon la technique de Green fournie par un laboratoire américain (des essais sont en cours avec d'autres laboratoires). L'indication de ces cultures est limitée aux malades qui ne pourraient pas survivre sans l'utilisation de ce type de techniques : patients brûlés sur plus de $70 \%$ de la surface corporelle totale, $60 \%$ de la surface corporelle totale étant en $3^{\mathrm{e}}$ degré. Quarante-huit patients ont été aujourd'hui recouverts par cette technique, 5 sont morts, 43 ont survécus alors que leur probabilité de survie était de l'ordre de $10 \%$. Cette indication comme couverture définitive est la seule que nous utilisions depuis 1991. Elle nécessite des cultures de grande qualité capables de se multiplier et de survivre in vivo.

L'utilisation de cultures comme inducteur, activateur de la cicatrisation procède d'une autre philosophie et ne nécessite pas la même qualité de produit. Une plaie recouverte de cultures cicatrise plus rapidement, certaines plaies chroniques guérissent après application de cultures de peau. Il ne s'agit en aucun cas de la prolifération des cellules de la culture qui disparaissent mais de la cicatrisation des tissus du receveur. L'hypothèse retenue à ce phénomène est celle de la fourniture par ces cellules vivantes de facteurs de croissance en quantité harmonieuse.

L'activation de la cicatrisation est un phénomène intéressant que l'on peut utiliser dans le traitement des brûlures superficielles, des zones de prise de greffe. La mise en suspension de kératinocytes dans de la colle biologique ou l'utilisation de lysats de kératinocytes en spray ont été proposés dans la même indication.

\section{Questions soulevées par les brûlures radiologiques}

Notre expérience des brûlures radiologiques est courte et il faut bien avouer qu'elle nous pose plus de questions qu'elle ne nous offre de solutions. Il faut immédiatement ajouter que les solutions attendues ne se trouvent pas non plus dans la littérature scientifique traitant de la question et qu'il n'y a certainement pas un mode unique de recouvrement de ces lésions mais plutôt des choix effectués cas 
par cas et basés sur une parfaite connaissance des possibilités offertes par l'état actuel de la médecine.

Quels sont les problèmes posés ?

\subsection{Sur le plan général}

Le brûlé radiologique tout comme le brûlé thermique est un agressé qui présente une plaie plus ou moins étendue, en surface comme en profondeur, à l'origine d'une réaction inflammatoire générale et d'un risque infectieux volontiers majoré par une irradiation générale. La douleur générée par les lésions cutanées est très particulière, elle est permanente, intense partiellement liée seulement à des réactions nociceptives et donc difficilement calmée par la morphine. Cette douleur est un parfait témoin des lésions de radionécrose en cours ou à venir.

La brûlure radiologique, dans les cas rencontrés, n'a jamais été cause de choc immédiat comme le sont les brûlures thermiques mais, chez l'un de nos malades, nous avons observé un choc d'aspect septique sans germe isolé à la troisième semaine d'évolution alors qu'apparaissait une épithélite exsudative de grande surface.

\subsection{Sur le plan local}

La classification des brûlures non radiologiques est basée sur l'observation histologique concomitante : on parle de brûlures superficielles (cicatrisation spontanée) lorsque la brûlure est purement épidermique ou lorsqu'elle écrête la membrane basale n'atteignant que très superficiellement le derme; on parle de brûlures profondes (nécessité de greffes) lorsque la totalité de l'épiderme et de la membrane basale est détruite, la brûlure dermique est totale ; on parle de brûlures intermédiaires (cicatrisation aléatoire) lorsque persistent pour seul épiderme les follicules pileux, la brûlure dermique est profonde.

La brûlure radiologique est classifiée en érythème, épidermite sèche, épidermite exsudative et nécrose par vascularité. Il est tentant pour le spécialiste des brûlures de faire un amalgame entre érythème et premier degré, épidermite et brûlures superficielles, nécrose et brûlures profondes : l'expérience prouve qu'il n’en est rien et que le génie évolutif des brûlures radiologiques est très particulier.

L'épidermite exsudative nous semble un passage obligé vers des lésions de nécrose, son aspect est faussement superficiel.

Lorsqu' une cicatrisation est obtenue, elle est fragile, lyse facilement et l'aspect est alors celui d'une plaie inhomogène avec des ulcérations couvertes de tâches 
de bougie (épidermisation à partir des follicules pileux) jouxtant des zones cicatrisées.

Lorsqu'il existe des lésions musculaires, elles prennent l'aspect d'un tissu sec, noir ou brun. L'excision permet de retrouver un socle théoriquement sain mais, au pansement suivant le même aspect de nécrose réapparaît.

Enfin, de façon totalement inopinée, alors qu'une greffe a été mise en place et est bien intégrée, il apparaît une nécrose sous-jacente...

\section{Réflexions sur la prise en charge des radionécroses}

\subsection{Sur le plan général}

Il est très important d'insister sur cet aspect volontiers méconnu de la thérapeutique. Le traitement local n'est possible que sur un malade en bon équilibre nutritionnel.

Il existe un certain nombre d'impératifs thérapeutiques :

- le confort du patient doit être assuré. Il s'agit du confort d'installation qui peut exiger différents types de supports du simple matelas mousse au lit fluide. Il s'agit également d'assurer la sédation à l'aide de morphine sous toutes ses formes (orale, parentérale en particulier sous forme de PCA, intrathécale) mais aussi de neuroleptiques, d'antiépileptiques ou de benzodiazépines actifs sur les douleurs de déafférentation;

- la prévention de l'infection doit être une préoccupation de tous les instants ;

- un programme de nutrition adapté aux dépenses caloriques doit être mis en place qui ressemble à celui institué dans la brûlure grave. Ce programme doit n’être que très peu interrompu par les différents gestes chirurgicaux sous anesthésie pratiqués ;

- en dehors de problèmes généraux septiques ou d'irradiation, les antibiothérapies systémiques n'ont pas d'indication. L'infection locale se traite par des soins locaux si nous ne voulons pas aboutir aux résistances bactériennes auxquelles nous avons été confrontés.

\subsection{Sur le plan local}

\section{Au stade de l'épidermite et des phlyctènes}

Le traitement local est celui d'une brûlure superficielle : pansement quotidien après lavage par des antiseptiques de type chlorexidine par un topique 
anti-infectieux de type silver-sulfadiazine. Il faut, à ce stade éviter la colonisation et permettre la cicatrisation. L'utilisation de facteurs de croissance sous la forme de cultures cellulaires ou de produits type Transcyte est sans doute utile.

Les lésions de ce type qui n'ont pas guéri au bout d'un mois doivent être greffées par des autogreffes expansées. La greffe doit être précédée d'une excision des résidus dermiques qui sont, a priori, suspects. Il est donc intéressant pour ne pas greffer sur la fascia musculaire de passer par une étape de derme artificiel (Integra). L'emplacement des prises de greffe doit avoir été soigneusement discuté afin d'éviter toutes les zones irradiées même si leur aspect est sain.

\section{Au stade de la nécrose}

L'excision des tissus irradiés évoluant vers la nécrose est impérative : le problème est celui de l'heure et de l'étendue du geste qui reste à déterminer en fonction de la reconstitution dosimétrique et de l'aspect macroscopique.

Toute excision doit être suivie d'une couverture temporaire par une xéno- ou une allogreffe. L'immunodépression induite par une irradiation concomitante permettra une survie plus longue de ces allogreffes.

La couverture définitive des lésions par lambeaux ou par autogreffes ne doit être réalisée que lorsqu'on est sûr de la non-évolutivité du socle receveur. L'utilisation de la peau artificielle Integra permet d'attendre et de greffer sur un socle de qualité.

L'indication des cultures cellulaires dans le traitement des lésions radiologiques est difficile à envisager dans l'état actuel. L'effet activateur de la cicatrisation de ces cultures peut cependant être essayé dans les lésions où il semble encore exister des éléments épidermiques viables : c'est probablement la manière la plus harmonieuse de délivrer des facteurs de croissance locaux.

\section{Conclusion}

L'évolution des brûlures radiologiques reste encore un mystère et il est donc impossible de dégager une conduite à tenir logique des quelques cas déjà traités. L'habitude de prise en charge des grands dégâts cutanés dans les centres de traitement des brûlés en fait un lieu privilégié pour le traitement de ces lésions lorsqu'elles sont graves. La précocité de l'action chirurgicale, la prévention de l'infection, l'utilisation de substituts cutanés temporaires pourraient être la clé du succès. 


\section{MOYENS DE RECOUVREMENT DU BRÛLÉ GRAVE}

\section{RÉFÉRENCES}

Bell E., Erlich H.P., Sher S. ef al. (1981) Development and use of a living skin equivalent, Plast. Reconstr. Surg. 67, 386-392.

Berthot F., Damour O. (1997) In vitro reconstructed skin models for wound coverage in deep burns, Br. I. Dermatol. 136, 809-816.

Boyce S.T.. Gorestsky M.J., Greenhalgh D.G. et al. (1995) Comparative assessment of cultured skin substitutes and native skin autograft for treatment of full-thickness burns, Ann. Surg. 222, 743752.

Burke J.F., Yannas I.V., Quinby W.C. et al. (1981) Sucessful use of a physiologically acceptable artificial skin in the treatment of extensive burn injury, Ann. Surg. 194, 413-428.

Carsin H., Ainaud P., Le Béver H. et al. (1997) Objectifs, résultats et perspectives du traitement des brûlés en 1997, Bull. Acad. Natl. Méd. 181. 1307-1320.

Coulomb B., Friteau L., Baruch J. et al. (1998) Advantages of the presence of living dermal fibroblasts within in vitro reconstructed skin for grafting in humans, Plast. Reconstr. Surg. 101, 1891-1903.

De Luca M., Albanese E., Bondanza S. et al. (1989) Multicenter experience in the treatment of burns with autologous and allogenis cultures apithelium. fresh or preserved in a frozen state, Burns $\mathbf{1 5}$, 303-309.

Duislaeger L., Delaey B., Vanderkelen A. (1998) Short and long-term results of application of allogeneic cultured keratinocytes on burn wounds and burn scar, Eur. J. Plast. Surg. 21, 14-18.

Frank D.H., Watchel T., Frank H.A. et al. (1983) Comparison of Biobrane. porcine and human allograft as biologic dressings for burn wounds, J. Bum Care Rehabil. 4, 186-190.

Heimbach D., Luterman A., Burke J.F. et al. (1988) Artificial dermis for major burns: a multi-center randomized clinical trial, Ann. Surg. 208, 31,3-320.

Hickerson W.L., Compton C.C., Fletchall S. (1994) Cultured epidermal autograft and allodermis combination for permanent burn wound coverage. Burns 1, S52-S56.

Moll I., Houdek P., Schmidt H. et al. (1998) Characterization of epidermal wound healing in a human skin organ culture model: acceleration by transplanted keratinocytes, J. Investig. Dermat. 111 251-258.

Nunez-Gutierrez H., Castro-Munozledo F., Kuri-Harcuch W. (1996) Combined use of allograft and autograft epidermal cultures in therapy of burns, Plast. Reconstr. Surg. 98, 929-939.

Pandya A.N., Woodward B., Parkhouse N. (1998) The use of cultured autologous keratinocytes with Integra in the resurfacing of acute burns, Plast. Reconstr. Surg. 102, 825-828.

Pruitt B.A. (1997) The evolutionary of biologic dressings and skin substitutes, J. Burn Care Rehabil. 18, S2-S5.

Rheinwald J.G., Green H. (1975) Serial cultivation of strains of human epidermal keratinocytes: the formation of keratinizing colonies from single cells, Cell. 6, 33I-344.

Sabolinski M.L., Alvarez O., Auletta M. et al. (1996) Cultured skin as a smart material for healing wounds: experience in venous ulcers, Biomaterials 17, 311-320.

Sheridan R.L., Thompkins R.G. (1995) Cultured autologous epithelium in patients with burns of ninety or more of the body surface, J. Trauma $38,48-50$.

Stark G.B., Kaiser H.W., Kopp J. et al. (1995) Cultured autologous keratinocyles suspended in fibrin glue with allogenic overgraft for definitive wouns coverage. A case report, Eur. J. Plast. Surg. 18. 267-27I.

Wainwright D., Madden M., Luterman A. et al. (1996) Clinical evaluation of An Accllular Allograft Dermal Matrix in full-Thickness burns, J. Burn Care Rehabil. 17. 124-136.

Yang C.C., Shih T.S., Chu T.A. et al. (1980) The intermingled transplantation of auto and homografts in severe burns, Burns 6, 141-145. 